PROCEEDINGS OF THE

AMERICAN MATHEMATICAL SOCIETY

Volume 138, Number 4, April 2010, Pages 1479-1489

S 0002-9939(09)10197-1

Article electronically published on November 18, 2009

\title{
REGULARITY OF \\ A FRACTIONAL PARTIAL DIFFERENTIAL EQUATION DRIVEN BY SPACE-TIME WHITE NOISE
}

\author{
MIN NIU AND BIN XIE \\ (Communicated by Walter Craig)
}

\begin{abstract}
We will deal with one dimensional stochastic fractional order partial differential equation driven by space-time white noise. The existence and uniqueness of the solution and especially some regularities of the solution are investigated. The regularities of the solution in its time and space variables depend on the relation of the fractional order of its operator and coefficients.
\end{abstract}

\section{INTRODUCTION}

Stochastic fractional order partial differential equations (driven by Gaussian noise or not) have recently been studied by some authors. In fact, these kinds of equations can be widely used in physics, fractal medium, quantum fields, risk management and other fields; see [4, 9, 12.

This paper aims to give a deep study of the work introduced by L. Debbi and M. Dozzi in 2005 [4. In their work, they generalized the stochastic heat equation in the framework of J. B. Walsh [13] to a stochastic fractional partial differential equation with respect to a fractional differential operator $\mathfrak{D}_{\alpha}$ defined as below, which was recently introduced by L. Debbi 3 . A differential operator $\mathfrak{D}_{\alpha}$ is called a fractional differential operator with parameters $\alpha, \delta$ if $\mathfrak{D}_{\alpha}$ is defined by

$$
\mathfrak{D}_{\alpha}^{\delta} \phi(x):=\mathfrak{D}_{\alpha} \phi(x)=\mathfrak{F}^{-1}\left\{-|\cdot|^{\alpha} e^{-i \delta \pi \operatorname{sgn}(\cdot) / 2} \mathfrak{F}(\phi)\right\}(x),
$$

where $\delta$ is less than or equal to the minimum of $\alpha-[\alpha], 2+[\alpha]_{2}-\alpha,[\alpha]$ and $[\alpha]_{2}$ denote the largest integer and largest even integer less than or equal to $\alpha$, and $\mathfrak{F}$ and $\mathfrak{F}^{-1}$ are the Fourier and Fourier inverse transformations respectively. This operator is a generalization of various well-known operators, such as the Laplacian operator, the inverse of the generalized Riesz-Feller potential, the Riemann-Liouville differential operator; see [3, 4, 8. For simplicity of notation, the parameter $\delta$ will be omitted in this paper if there is no confusion.

Received by the editors June 3, 2009.

2010 Mathematics Subject Classification. Primary 60H15; Secondary 26A33, 35R60.

Key words and phrases. Space-time white noise, fractional differential operator, regularity, Hölder continuity, strong differentiability.

The first author was supported in part by the National Natural Science Foundation of China under grant No. 10871202.

The second author was supported in part by the Grant-in-Aid for young scientists (start-up) 20840019 (JSPS) and Grant-in-Aid for young scientists (B) 21740067(MEXT).

(C)2009 American Mathematical Society Reverts to public domain 28 years from publication 
More precisely, the following fractional partial differential equation driven by space-time white noise in one dimensional space will be studied:

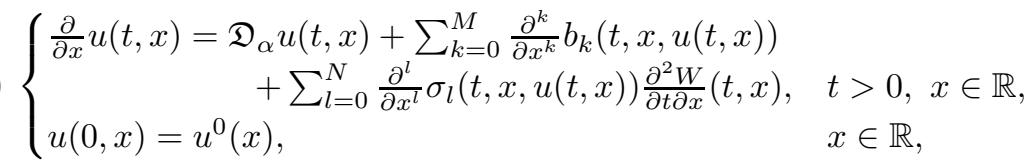

where $M, N$ are two integers and the coefficients $b_{k}$ and $\sigma_{l}$ are some measurable functions defined on $[0, \infty) \times \mathbb{R} \times \mathbb{R}$ and $W(t, x)$ is a Brownian sheet on $[0, \infty) \times \mathbb{R}$,

(see [13]) $\left(\frac{\partial^{2} W}{\partial t \partial x}(t, x)\right.$ is usually called a space-time white noise), and $u^{0}$ is a random function defined on $\mathbb{R}$. Throughout this paper, we will assume that $\alpha \in(1, \infty) / \mathbb{N}$.

Our interests are twofold. One is to study the fundamental problem, the existence and uniqueness of the solution, under some conditions on $M$ and $N$. L. Debbi and M. Dozzi studied only the special case for $N=0$. However, we are interested in the more general case (1.1). The other is to discuss the regularities in time and space variables of the solution $u(t, x)$ according to the relation of $\alpha, M$ and $N$. Although some stochastic fractional order partial differential equations are studied as we said, there are few authors who pay attention to the regularities of solutions.

The regularities of the solution of an even order (larger than 2) stochastic partial differential equation driven by cylindrical Brownian motion with uniformly bounded Lipschitz drift coefficients and uniformly bounded constant diffusion coefficients were initially studied by T. Funaki [5, 6] and then were restudied in [1]. Relative results will be generalized to all fractional order $\alpha>1$ in this paper. Recall that $\mathfrak{D}_{\alpha}$ is just the Laplacian operator when $\alpha=2$. For the regularities of solutions of stochastic heat equations with space-time white noise as their driving noises, we also refer readers to $11,13,14$. The Hölder continuity of the solution $u(t, x)$ is also studied by L. Debbi and M. Dozzi. However, in their framework, although under the strict assumption $N=0, \alpha<3$ is assumed to guarantee that the solution $u(t, x)$ has a Hölder continuous version in its space variable. It is our main interest to release their restriction on $\alpha$. In fact, we will show that for all $\alpha>1$, the Hölder continuity of the solution in the space variable will be fulfilled; see Theorem 2.5] in Section 2 ,

The organization of this paper is as follows. In the next section, we first recall some basic facts and then formulate our main results of this paper. The proofs of the main results will be completed in the last section.

\section{Foundation AND MAin Result}

We first give the mathematical meaning of the stochastic fractional partial differential equation (1.1). Here we give two kinds of definitions of the solution according to that introduced initially by J. B. Walsh [13]; see also [2, 7, 10].

Let $(\Omega, \mathcal{F}, \mathbb{P})$ be a completed probability space and let $\{W(t, x), t \geq 0, x \in \mathbb{R}\}$ be a Brownian sheet on this probability space. Define the filtration $\mathcal{F}_{t}$ by

$$
\mathcal{F}_{t}=\sigma\{W(s, x): 0 \leq s \leq t, x \in \mathbb{R}\} .
$$

Then $\{W(t, x), t \geq 0, x \in \mathbb{R}\}$ generates a continuous orthogonal martingale measure in the sense of J. B. Walsh [13. Assume that $g_{\alpha}(t, x)$ is the Green function 
corresponding to (1.1), i.e., the fundamental solution of the Cauchy problem:

$$
\left\{\begin{array}{l}
\frac{\partial}{\partial t} g_{\alpha}(t, x)=\mathfrak{D}_{\alpha} g_{\alpha}(t, x), \\
g_{\alpha}(0, x)=\delta_{0}(x)
\end{array}\right.
$$

where $\delta_{0}$ denotes the Dirac function at the point 0 . Then $g_{\alpha}(t, x)$ can be expressed using Fourier analysis:

$$
g_{\alpha}(t, x)=\frac{1}{2 \pi} \int_{\mathbb{R}} \exp \left\{-i \xi x-t|\xi|^{\alpha} e^{-i \delta \pi \operatorname{sgn}(\xi) / 2}\right\} d \xi,
$$

which is in general nonsymmetric in $x$ and may be negative. But it still has some good properties; for example, it satisfies the Chapman-Kolmogorov equation and is smooth in $x$ for each fixed $t>0$, and so on. Hereafter we assume that the initial datum $u^{0}$ of (1.1) is $\mathcal{F}_{0}$-measurable. Now let us illustrate the definitions of the solution of (1.1).

Definition 2.1. (I) An $\mathcal{F}_{t}$-adapted random field $\{u(t, x), t \geq 0, x \in \mathbb{R}\}$ is said to be a mild solution of (1.1) with initial value $u^{0}$ if the following integral equation is fulfilled:

$$
\begin{aligned}
u(t, x) & =\int_{\mathbb{R}} g_{\alpha}(t, x-y) u^{0}(y) d y \\
& +\sum_{k=0}^{M}(-1)^{k} \int_{0}^{t} \int_{\mathbb{R}} \frac{\partial^{k}}{\partial y^{k}} g_{\alpha}(t-s, x-y) b_{k}(s, y, u(s, y)) d s d y \\
& +\sum_{l=0}^{N}(-1)^{l} \int_{0}^{t} \int_{\mathbb{R}} \frac{\partial^{l}}{\partial y^{l}} g_{\alpha}(t-s, x-y) \sigma_{l}(s, y, u(s, y)) W(d s, d y) \text { a.s. }
\end{aligned}
$$

where $g_{\alpha}(t, x)$ denotes the Green function determined by (2.1) and the stochastic integral with respect to $W(t, x)$ is understood in the sense of that introduced by J. B. Walsh [13].

(II) An $\mathcal{F}_{t}$-adapted random field $\{u(t, x), t \geq 0, x \in \mathbb{R}\}$ is called a weak solution of (1.1) if for any test function $\varphi \in C_{0}^{\infty}(\mathbb{R})$, the following holds:

$$
\begin{aligned}
\int_{\mathbb{R}} u(t, x) \varphi(x) d x & =\int_{\mathbb{R}} u^{0}(x) \varphi(x) d x+\int_{0}^{t} \int_{\mathbb{R}} u(s, x) \mathfrak{D}_{\alpha}^{*} \varphi(x) d s d x \\
& +\sum_{k=0}^{M}(-1)^{k} \int_{0}^{t} \int_{\mathbb{R}} b_{k}(s, x, u(s, x)) \varphi^{(k)}(x) d s d x \\
& +\sum_{l=0}^{N}(-1)^{l} \int_{0}^{t} \int_{\mathbb{R}} \sigma_{l}(s, x, u(s, x)) \varphi^{(l)}(x) W(d s, d x) \text { a.s. }
\end{aligned}
$$

where $\mathfrak{D}_{\alpha}^{*}$ denotes the dual operator of $\mathfrak{D}_{\alpha}, C_{0}^{\infty}(\mathbb{R})$ denotes the collection of all smooth functions with compact supports on $\mathbb{R}$ and $\varphi^{(k)}(x)$ denotes the $k$-th derivative, $k=0,1,2, \ldots$.

(III) We say that the uniqueness of the mild (weak) solution of (1.1) holds if for any two mild (weak) solutions $u(t, x)$ and $\tilde{u}(t, x)$ of (1.1), we have that $u(t, x)=$ $\tilde{u}(t, x)$ a.s., for all $t \geq 0, x \in \mathbb{R}$.

In the above definitions, it is natural to require that all terms appearing in (2.2) and (2.3) are well defined. In fact, this can be satisfied under the following Hypothesis $\mathbf{H}$; see Theorem 2.3 below: 
(H1) $M=[\alpha]$ and $N=\left[\frac{\alpha-1}{2}\right]$.

(H2) The drift and diffusion coefficients satisfy globally Lipschitz continuous conditions in their third variables; i.e., there exists a generic positive constant $C$ such that

$$
\sum_{k=0}^{M}\left|b_{k}(t, x, z)-b_{k}(t, x, \tilde{z})\right|+\sum_{l=0}^{N}\left|\sigma_{l}(t, x, z)-\sigma_{l}(t, x, \tilde{z})\right| \leq C|z-\tilde{z}|, z, \tilde{z} \in \mathbb{R} .
$$

It is clear that from the hypothesis $(\mathbf{H} 2)$ the uniform linear growth condition of the drift and diffusion coefficients in their third variables is satisfied. In fact, we mean that there exists a constant $C$ such that

$$
\sum_{k=0}^{M}\left|b_{k}(t, x, z)\right|+\sum_{l=0}^{N}\left|\sigma_{l}(t, x, z)\right| \leq C(1+|z|), z \in \mathbb{R} .
$$

For the relation of weak solution and mild solution, the following theorem can be formulated under our hypothesis.

Theorem 2.2. Under Hypothesis $\mathbf{H}$, a weak solution of the stochastic fractional partial differential equation (1.1) is equivalent to its mild solution.

Proof. The proof can be accomplished by the stochastic Fubini Theorem relative to the stochastic integral of orthogonal martingale measures, approximation and the semigroup properties corresponding to $g_{\alpha}(t, x)$ as usual; see [2, 7, 13] for obvious modifications. Here the detailed explanations of the proof will be omitted.

In the original paper 4, only the definition of a weak solution is introduced; however, the mild solution is not mentioned. The above theorem gives the clear relation between weak solutions and mild solutions of (1.1). In the following, let $T$ denote a fixed positive time and let $B_{T, p}(p \geq 2)$ denote the class of all $\mathcal{F}_{t}$-adapted random fields $\{u(t, x),(t, x) \in[0, T] \times \mathbb{R}\}$ satisfying

$$
\sup _{(t, x) \in[0, T] \times \mathbb{R}} \mathbb{E}\left[|u(t, x)|^{p}\right]<\infty .
$$

It is clear that for each fixed $T$ and $p, B_{T, p}$ is a Banach space. For simplicity of notation, we write $B_{T}$ for $B_{T, p}$ when $p=2$. We say that the random variables $u^{0}(x)$ with index $x \in \mathbb{R}$ are $L^{p}(\Omega)$-bounded, $p \geq 2$, if

$$
\sup _{x \in \mathbb{R}} \mathbb{E}\left[\left|u^{0}(x)\right|^{p}\right]<\infty .
$$

Now we can show that Hypothesis $\mathbf{H}$ implies the existence and uniqueness of a solution of (1.1). More precisely, the following holds:

Theorem 2.3. If Hypothesis $\mathbf{H}$ is satisfied and the initial datum $u^{0}$ is $L^{2}(\Omega)$ bounded, then there exists a solution $u(t, x)$ such that $u \in B_{T}$ and the uniqueness holds. Moreover if the initial datum $u^{0}$ is $L^{p}(\Omega)$-bounded, $p>2$, then we have that the solution $u \in B_{T, p}$.

Remark 2.4. (i) It is clear that the solution of (1.1) depends on the parameters $\alpha, \delta$ of the fractional operator $\mathcal{D}_{\alpha}$ and $M, N$ appearing in our equation. However, for simplicity, we will write $u(t, x)$ for the solution of (1.1) throughout this paper if it is not necessary for us to express these parameters. 
(ii) The above theorem is also fulfilled for $\alpha=2 m, m \in \mathbb{N}$, which was initially studied by T. Funaki [6]. It is well known that conservative and nonconservative Ginzburg-Landau equations are important examples. We extend Funaki's result from an even-order differential operator to the fractional case.

The following is the key theorem of this paper, which deals with the regularities of the solution $u(t, x)$ of (1.1). We will show that under Hypothesis $\mathbf{H}$, the solution $u(t, x)$ has a Hölder continuous version both in time $t$ and space $x$. Our result removes the restriction of $\alpha$ used in Theorem 2 [4 for the regularity in the space variable. Before stating our main results, let us introduce some notation for simplicity. Define

$$
B_{\alpha, k}(t, x)=\int_{0}^{t} \int_{\mathbb{R}} \frac{\partial^{k}}{\partial y^{k}} g_{\alpha}(t-s, x-y) b_{k}(s, y, u(s, y)) d s d y, k=0, \cdots, M,
$$

and

$$
S_{\alpha, l}(t, x)=\int_{0}^{t} \int_{\mathbb{R}} \frac{\partial^{l}}{\partial y^{l}} g_{\alpha}(t-s, x-y) \sigma_{l}(s, y, u(s, y)) W(d s, d y), l=0, \cdots, N .
$$

Now we can formulate the main results relative to the regularities of the solution $u(t, x)$ of (1.1). In some sense, the strong differentiability of $B_{\alpha, k}(t, x)$ and $S_{\alpha, l}(t, x)$ in $x$ is also established.

Theorem 2.5. Under Hypothesis $\mathbf{H}$, for all $\alpha>1$, there exists a continuous version for the solution $u(t, x)$ of (1.1) which is even Hölder continuous both in $t$ and $x$. More precisely, for large enough $p$ and each $T>0$, the following estimates hold:

(1) For each $i$ with $k+i=M$, there exists a positive constant $C$ such that for all small enough $\varepsilon_{0}>0$ the following holds:

$$
\mathbb{E}\left[\left|\frac{\partial^{i}}{\partial x^{i}} B_{\alpha, k}(t, x)-\frac{\partial^{i}}{\partial z^{i}} B_{\alpha, k}(t, z)\right|^{p}\right] \leq C|x-z|^{(p-1)(\alpha-M)-\varepsilon_{0}} .
$$

(2) For each $j$ with $l+j=N$, there exists a positive constant $C$ such that for all small enough $\varepsilon_{0}>0$ the following holds:

$$
\mathbb{E}\left[\left|\frac{\partial^{j}}{\partial x^{j}} S_{\alpha, l}(t, x)-\frac{\partial^{j}}{\partial z^{j}} S_{\alpha, l}(t, z)\right|^{p}\right] \leq C|x-z|^{\left(p-\frac{1}{2}\right)\left(\frac{\alpha-1}{2}-N\right)-\varepsilon_{0}} .
$$

(3) For all $i, j$ satisfying $k+i \leq M$ and $l+j \leq N$ and any $t, t^{\prime} \in[0, T]$, there exists a constant $C$ such that

$$
\mathbb{E}\left[\left|\frac{\partial^{i}}{\partial x^{i}} B_{\alpha, k}(t, x)-\frac{\partial^{i}}{\partial x^{i}} B_{\alpha, k}\left(t^{\prime}, x\right)\right|^{p}\right] \leq C\left|t-t^{\prime}\right|^{\frac{p(\alpha-k-i)}{\alpha}}
$$

and

$$
\mathbb{E}\left[\left|\frac{\partial^{j}}{\partial x^{j}} S_{\alpha, l}(t, x)-\frac{\partial^{j}}{\partial x^{j}} S_{\alpha, l}\left(t^{\prime}, x\right)\right|^{p}\right] \leq C\left|t-t^{\prime}\right| \frac{p(\alpha-2(l+j)-1)}{2 \alpha} .
$$

Remark 2.6. (i) By the Kolmogorov regularization theorem, the estimates (2.4) and (2.5) imply that $B_{\alpha, k}(t, x)$ and $S_{\alpha, l}(t, x)$ have modifications in $\mathcal{C}^{\alpha-k-\varepsilon_{0}}(\mathbb{R})$ and $\mathcal{C}^{\frac{\alpha-1}{2}-l-\varepsilon_{0}}(\mathbb{R})$ respectively for any small enough $\varepsilon_{0}$ and each $k \leq M, l \leq N$, where for each positive noninteger $\lambda, \mathcal{C}^{\lambda}(\mathbb{R})$ denotes the family of all functions $f \in \mathcal{C}^{[\lambda]}(\mathbb{R})$ with Hölder continuous derivative $f^{[\lambda]}$ of order $\lambda-[\lambda]$. In particular, for all $\alpha>1$ 
and each $l \leq N$ the stochastic convolution $S_{\alpha, l}(t, x)$ has a Hölder continuous version of order

$$
\frac{\alpha-1}{2 \alpha}-\left[\frac{\alpha-1}{2 \alpha}\right]-\varepsilon_{0}
$$

for all small enough $\varepsilon_{0}$ in $x$.

(ii) Under Hypothesis $\mathbf{H}$, from (2.6) and (2.7), it follows easily that for any small enough $\varepsilon_{0}, B_{\alpha, k}(t, x)$ and $S_{\alpha, l}(t, x)$ have Hölder continuous versions in time $t$ of orders

$$
\frac{\alpha-k}{\alpha}-\varepsilon_{0}
$$

and

$$
\frac{\alpha-2 l-1}{2 \alpha}-\varepsilon_{0}
$$

respectively. Therefore, combining with $(i)$, if Hypothesis $\mathbf{H}$ is fulfilled, then we can conclude that the solution $u(t, x)$ of (1.1) has a $(\beta, \tilde{\beta})$-Hölder continuous version both in $t$ and $x$ for any

$$
\beta<\min \left\{\frac{\alpha-M}{\alpha}, \frac{\alpha-2 N-1}{2 \alpha}\right\}
$$

and

$$
\tilde{\beta}<\min \left\{\alpha-M, \frac{\alpha-1}{2}-N\right\} .
$$

In other words, we know that $u(t, x)$ is $\beta$-Hölder and $\tilde{\beta}$-Hölder continuous in its time variable $t$ and space variable $x$ respectively.

\section{Proof of MAIn Theorem}

For the convenience of the reader, we first summarize some elementary properties of the kernel $g_{\alpha}(t, x)$ according to Lemma 1 and Corollaries 1 and 2 in [4] as below.

Lemma 3.1. The following holds:

(1) (Generalized scaling properties) For any $k \geq 1$,

$$
\frac{\partial^{k}}{\partial x^{k}} g_{\alpha}(t, x)=t^{-\frac{k+1}{\alpha}} \frac{\partial^{k}}{\partial x^{k}} g_{\alpha}\left(1, t^{-\frac{1}{\alpha}} x\right) .
$$

(2) For each $\alpha$, there exists a positive constant $C$ such that

$$
\left|\frac{\partial^{k}}{\partial x^{k}} g_{\alpha}(1, x)\right| \leq C \frac{1+|x|^{\alpha+k-1}}{\left(1+|x|^{\alpha+k}\right)^{2}},
$$

and for $p \in\left(\frac{1}{\alpha+k+1}, \frac{\alpha+1}{k+1}\right)$,

$$
\int_{0}^{T} \int_{\mathbb{R}}\left|\frac{\partial^{k}}{\partial x^{k}} g_{\alpha}(t, x)\right|^{p} d t d x<\infty .
$$

Now we start to show our main results formulated in Section 2. We are first going to show the existence and uniqueness of the solution of (1.1).

Proof of Theorem 2.3. This theorem can be easily proved by applying the fixed point theorem or successive approximation; see [4, 13. We will adapt the successive approximation method here as in [4]. As we know, the main difference between our assumption and that of L. Debbi and M. Dozzi [4 is in the diffusion coefficients. Therefore it is enough for us to write down the outline of the proof and fill in some needed parts for our case. More precisely, we will give some explanations only for 
the case corresponding to stochastic convolution parts in (2.2). Define the sequence $\left\{u_{n}(t, x)\right\}_{n=0}^{\infty}$ of successive approximations as below:

$$
\begin{aligned}
u_{0}(t, x) & =\int_{\mathbb{R}} g_{\alpha}(t, x-y) u^{0}(y) d y \\
u_{n+1}(t, x) & =u_{0}(t, x)+\sum_{k=0}^{M}(-1)^{k} \int_{0}^{t} \int_{\mathbb{R}} \frac{\partial^{k}}{\partial y^{k}} g_{\alpha}(t-s, x-y) b_{k}\left(s, y, u_{n}(s, y)\right) d s d y \\
& +\sum_{l=0}^{N}(-1)^{l} \int_{0}^{t} \int_{\mathbb{R}} \frac{\partial^{l}}{\partial y^{l}} g_{\alpha}(t-s, x-y) \sigma_{l}\left(s, y, u_{n}(s, y)\right) W(d s, d y) .
\end{aligned}
$$

To construct a solution, it is enough to show that $\left\{u_{n}(t, x)\right\}_{n=0}^{\infty}$ is a Cauchy sequence in $B_{T}$. We are first going to prove that for each $n, u_{n}(t, x) \in B_{T}$ by induction if $u^{0}$ is $L^{2}(\Omega)$-bounded. It is clear that it holds for $n=0$. Now we assume that our claim holds for some $n \geq 1$. Noting that for each $l \leq N$,

$$
2<\frac{\alpha+1}{l+1}
$$

and by (3.1) in Lemma 3.1 and $\mathbf{H}$, we can easily see that

$$
\begin{aligned}
& \mathbb{E}\left[\left|\int_{0}^{t} \int_{\mathbb{R}} \frac{\partial^{l}}{\partial y^{l}} g_{\alpha}(t-s, x-y) \sigma_{l}\left(s, y, u_{n}(s, y)\right) W(d s, d y)\right|^{2}\right] \\
\leq & \int_{0}^{t} \int_{\mathbb{R}}\left(\frac{\partial^{l}}{\partial y^{l}} g_{\alpha}(t-s, x-y)\right)^{2} \mathbb{E}\left[\sigma_{l}^{2}\left(s, y, u_{n}(s, y)\right)\right] d s d y \\
\leq & C\left(1+\sup _{(t, x) \in[0, T] \times \mathbb{R}} \mathbb{E}\left[\left|u_{n}(t, x)\right|^{2}\right]\right) \int_{0}^{t} \int_{\mathbb{R}}\left(\frac{\partial^{l}}{\partial y^{l}} g_{\alpha}(t-s, x-y)\right)^{2} d s d y \\
< & \infty .
\end{aligned}
$$

Hence by virtue of the corresponding proof of Theorem 1 in [4] and by our assumption, the proof of our claim can be completed easily. From now on, we will show that for each $l \leq N$, the following estimate holds:

$$
\begin{aligned}
& \mathbb{E}\left[\left|\int_{0}^{t} \int_{\mathbb{R}} \frac{\partial^{l}}{\partial y^{l}} g_{\alpha}(t-s, x-y)\left(\sigma_{l}\left(s, y, u_{n}(s, y)\right)-\sigma_{l}\left(s, y, u_{n-1}(s, y)\right)\right) W(d s, d y)\right|^{2}\right] \\
& \quad \leq C \int_{0}^{t}(t-s)^{-\frac{2 N+1}{\alpha}} H_{n-1}(s) d s,
\end{aligned}
$$

where

$$
H_{n}(s)=\sup _{x \in \mathbb{R}} \mathbb{E}\left[\left(u_{n+1}(s, x)-u_{n}(s, x)\right)^{2}\right] .
$$

By Lemma 3.1] it is easy to see that for any $s \leq T$, there exists a constant $C$ such that

$$
\sup _{x \in \mathbb{R}} \int_{\mathbb{R}}\left(\frac{\partial^{l}}{\partial y^{l}} g_{\alpha}(s, x-y)\right)^{2} d y \leq C s^{-\frac{2 l+1}{\alpha}}, l \leq N,
$$

and then we can obtain (3.2) by the Itô isometric formula and the Lipschitz condition on $\sigma_{l}$. Then using the relative estimates in the proof of Theorem 1 in [4], we 
can show that

$$
H_{n}(t) \leq C \int_{0}^{t}(t-s)^{-\max \left\{\frac{M}{\alpha}, \frac{2 N+1}{\alpha}\right\}} H_{n-1}(s) d s,
$$

which implies that $u_{n}(t, x)$ converges to $u(t, x)$ in $B_{T}$ in the same way as in Theorem 1 in 4 because of the hypothesis $(\mathbf{H} 1)$ and Lemma 3.3 in [13]. It is obvious that the limit $u(t, x)$ of the sequence $u_{n}(t, x)$ is a solution of (1.1). On the other hand, the uniqueness of the solution can be easily shown as usual, and the details will be omitted here.

In the following, the proof of Theorem 2.5 will be formulated in detail, which is based on [6] and is easier than the proof in [4].

Proof of Theorem 2.5. Owing to the Kolmogorov regularity theorem (see Corollary 1.2 in [13]), we can complete the proof of our main result by showing the estimates (2.4)-(2.7) respectively. However, the proofs for (2.6) and (2.7) can be easily accomplished (see Remark 3.2 below). Here we will consider the proofs only for (2.4) and (2.5). Recall that the first term

$$
\int_{\mathbb{R}} g_{\alpha}(t, x-y) u_{0}(y) d y
$$

in (2.2) used for the definition of a mild solution is a smooth function corresponding to $t$ and $x$ according to properties of $\mathcal{D}_{\alpha}$; see 4. Let us first show (2.5). In the following we assume $p \gg 1$. Let

$$
\kappa=\frac{\alpha}{2}-\left[\frac{\alpha-1}{2}\right]
$$

and

$$
\epsilon=(1+\alpha)(p-2)-\kappa .
$$

Then we see that $\kappa$ and $\epsilon$ are strictly positive, recalling that $\alpha$ is not an integer. By virtue of the Burkholder's inequality and the scaling property in Lemma 3.1 for each $j$ such that $l+j=N$, we have that

$$
\begin{aligned}
\mathbb{E}\left[\left|\frac{\partial^{j}}{\partial x^{j}} S_{\alpha, l}(t, x)-\frac{\partial^{j}}{\partial z^{j}} S_{\alpha, l}(t, z)\right|^{p}\right] \\
=\mathbb{E}\left[\left|\int_{0}^{t} \int_{\mathbb{R}} \frac{\partial^{N}}{\partial y^{N}}\left(g_{\alpha}(t-s, x-y)-g_{\alpha}(t-s, z-y)\right) \sigma_{l}(s, y, u(s, y)) W(d s, d y)\right|^{p}\right] \\
\leq C \mathbb{E}\left[\left|\int_{0}^{t} \int_{\mathbb{R}}\left(\frac{\partial^{N}}{\partial y^{N}}\left(g_{\alpha}(t-s, x-y)-g_{\alpha}(t-s, z-y)\right)\right)^{2} \sigma_{l}^{2}(s, y, u(s, y)) d s d y\right|^{p / 2}\right] \\
\leq C \mathbb{E}\left[\mid \int_{0}^{t} \int_{\mathbb{R}}(t-s)^{-\frac{2(N+1)}{\alpha}} \sigma_{l}^{2}(s, y, u(s, y))\right. \\
\left.\left.\cdot\left(\frac{\partial^{N}}{\partial y^{N}}\left(g_{\alpha}\left(1,(t-s)^{-\frac{1}{\alpha}}(x-y)\right)-g_{\alpha}\left(1,(t-s)^{-\frac{1}{\alpha}}(z-y)\right)\right)\right)^{2} d s d y\right|^{p / 2}\right],
\end{aligned}
$$


where $\frac{\partial}{\partial y} g_{\alpha}(t, x-y)=-\frac{\partial}{\partial x} g_{\alpha}(t, x-y)$ is used for the first equality. Hence by the Hölder's inequality and the above inequality, we obtain that

$$
\begin{aligned}
\mathbb{E} & {\left[\left|\frac{\partial^{j}}{\partial x^{j}} S_{\alpha, l}(t, x)-\frac{\partial^{j}}{\partial z^{j}} S_{\alpha, l}(t, z)\right|^{p}\right] } \\
\leq & C\left(\int_{0}^{t} \int_{\mathbb{R}} s^{-\frac{\epsilon q}{\alpha}}\left|\frac{\partial^{N}}{\partial y^{N}}\left(g_{\alpha}\left(1, s^{-\frac{1}{\alpha}}(x-y)\right)-g_{\alpha}\left(1, s^{-\frac{1}{\alpha}}(z-y)\right)\right)\right|^{(2-\gamma) q} d s d y\right)^{\frac{p}{2 q}} \\
& \times \int_{0}^{t} \int_{\mathbb{R}} s^{-\frac{(2(N+1)-\epsilon) p}{2 \alpha}}\left|\frac{\partial^{N}}{\partial y^{N}}\left(g_{\alpha}\left(1, s^{-\frac{1}{\alpha}}(x-y)\right)-g_{\alpha}\left(1, s^{-\frac{1}{\alpha}}(z-y)\right)\right)\right|^{\frac{\gamma p}{2}} d s d y, \\
:= & C I^{\frac{p}{2 q}} \times I I,
\end{aligned}
$$

where $q=\frac{p}{p-2}$ and $\gamma \in(0,2)$ is to be specified later; see (3.6). By a change of variables and Lemma 3.1 we can easily see that

$$
\begin{aligned}
I & \leq C \int_{0}^{t} \int_{\mathbb{R}} s^{-\frac{\epsilon q-1}{\alpha}}\left|\frac{\partial^{N}}{\partial y^{N}} g_{\alpha}(1, y)\right|^{(2-\gamma) q} d s d y \\
& \leq C \int_{0}^{t} s^{-\frac{\epsilon q-1}{\alpha}} d s<\infty .
\end{aligned}
$$

Recall that $\epsilon q<1+\alpha$ by the definition of $\epsilon$; see (3.3). Now we turn to estimate II. By the mean value theorem, we have that

$$
\begin{aligned}
& \frac{\partial^{N}}{\partial y^{N}}\left(g_{\alpha}\left(1, s^{-\frac{1}{\alpha}}(x-y)\right)-g_{\alpha}\left(1, s^{-\frac{1}{\alpha}}(z-y)\right)\right) \\
= & (x-z) \int_{0}^{1} s^{-\frac{1}{\alpha}} \frac{\partial^{N+1}}{\partial y^{N+1}} g_{\alpha}\left(1, s^{-\frac{1}{\alpha}}(\xi(\theta)-y)\right) d \theta,
\end{aligned}
$$

where $\xi(\theta)=x+\theta(x-z)$.

Therefore putting (3.5) into II and by a similar method to (3.4), we have that

$$
\begin{aligned}
\mathrm{II} & \leq C|x-z|^{\frac{\gamma p}{2}} \int_{0}^{t} \int_{\mathbb{R}} s^{-\frac{(2(N+1)-\epsilon+\gamma) p}{2 \alpha}}\left|\int_{0}^{1} \frac{\partial^{N+1}}{\partial y^{N+1}} g_{\alpha}\left(1, s^{-\frac{1}{\alpha}}(\xi(\theta)-y)\right) d \theta\right|^{\frac{\gamma p}{2}} d s d y, \\
& \leq C|x-z|^{\frac{\gamma p}{2}} \int_{0}^{t} \int_{\mathbb{R}} s^{-\frac{(2(N+1)-\epsilon+\gamma) p-2}{2 \alpha}}\left|\frac{\partial^{N+1}}{\partial y^{N+1}} g_{\alpha}(1, y)\right|^{\frac{\gamma p}{2}} d s d y, \\
& \leq C|x-z|^{\frac{\gamma p}{2}} \int_{0}^{t} s^{-\frac{(2(N+1)-\epsilon+\gamma) p-2}{2 \alpha}} d s .
\end{aligned}
$$

If

$$
\gamma<\frac{(2 p-1) \kappa}{p}
$$

then

$$
\frac{(2(N+1)-\epsilon+\gamma) p-2}{2 \alpha}<1 .
$$

Therefore, by the above estimate for II, we have that

$$
\text { II } \leq C|x-z|^{\frac{\gamma p}{2}},
$$

which implies the estimate (2.5) by the estimate (3.4) and by choosing an appropriate $\varepsilon_{0}$. 
The rest of this paper is devoted to proving (2.4). Let

$$
\kappa^{\prime}=\alpha-[\alpha]
$$

and assume that

$$
\beta<\min \left\{1, \frac{(p-1) \kappa^{\prime}}{p}\right\}
$$

Then as in the proof of (2.5), we can obtain that

$$
\begin{aligned}
\mathbb{E}[ & \left.\left|\frac{\partial^{i}}{\partial x^{i}} B_{\alpha, k}(t, x)-\frac{\partial^{i}}{\partial z^{i}} B_{\alpha, k}(t, z)\right|^{p}\right] \\
= & \mathbb{E}\left[\mid \int_{0}^{t} \int_{\mathbb{R}} \frac{\partial^{M}}{\partial y^{M}}(t-s)^{-\frac{M+1}{\alpha}} b_{k}(s, y, u(s, y))\right. \\
& \left.\left.\cdot\left(g_{\alpha}\left(1,(t-s)^{-\frac{1}{\alpha}}(x-y)\right)-g_{\alpha}\left(1,(t-s)^{-\frac{1}{\alpha}}(z-y)\right)\right) d s d y\right|^{p}\right] \\
\leq & C\left(\int_{0}^{t} \int_{\mathbb{R}} s^{-\frac{\epsilon^{\prime} q}{\alpha}}\left|\frac{\partial^{M}}{\partial y^{M}}\left(g_{\alpha}\left(1, s^{-\frac{1}{\alpha}}(x-y)\right)-g_{\alpha}\left(1, s^{-\frac{1}{\alpha}}(z-y)\right)\right)\right|^{(1-\beta) q} d s d y\right)^{\frac{p}{q}} \\
& \times \int_{0}^{t} \int_{\mathbb{R}} s^{-\frac{\left(M+1-\epsilon^{\prime}\right) p}{\alpha}}\left|\frac{\partial^{M}}{\partial y^{M}}\left(g_{\alpha}\left(1, s^{-\frac{1}{\alpha}}(x-y)\right)-g_{\alpha}\left(1, s^{-\frac{1}{\alpha}}(z-y)\right)\right)\right|^{\gamma p} d s d y \\
\leq & C|x-z|^{\beta p} \int_{0}^{t} s^{\frac{\epsilon^{\prime}-1}{\alpha}} d s \times \int_{0}^{t} s^{-\frac{\left(M+1-\epsilon^{\prime}+\beta\right) p-1}{\alpha}} d s<\infty,
\end{aligned}
$$

where $q$ satisfies $1 / p+1 / q=1$. Hence, we can choose small enough $\varepsilon$ such that (2.4) holds. Now the proof is completed.

Remark 3.2. (i) The estimates (2.6) and (2.7) can be established by the methods used in the above or by tracing the ways in [4]. However, only the special cases for $i=0$ and $j=0$ are considered in [4]. We generalized their results to the strong differentiability of $B_{\alpha, k}(t, x)$ and $S_{\alpha, l}(t, x)$ with respect to $x$.

(ii) Comparing to the method used in 4, we can see that our method is easier and only the integration on a bounded time interval is considered, which can be done for all $\alpha>1$ under our framework. However, in [4, to show the regularity, especially spatial regularity, the essential point is the following estimate:

$\int_{0}^{\infty} \int_{\mathbb{R}}\left|\frac{\partial^{k}}{\partial x^{k}} g_{\alpha}(t, 1+x)-\frac{\partial^{k}}{\partial x^{k}} g_{\alpha}(t, x)\right|^{p} d t d x<\infty, p \in\left(\frac{\alpha+1}{k+2}, \frac{\alpha+1}{k+1}\right), k<\alpha-1$, which gives the restriction of $\alpha<3$. In addition, we can also show that $S_{\alpha, l}(t, x)$ is the $j$-th differential in $x$ for all $j \leq\left[\frac{\alpha-1}{2}\right]-l$; see Remark [2.6.

\section{ACKNOWLEDGMENT}

The authors are very grateful to the referee for offering valuable suggestions to improve our manuscript.

\section{REFERENCES}

[1] C. Cardon-Weber, A. Millet, On strongly Petrovskii's parabolic SPDEs in arbitrary dimension and application to the stochastic Cahn-Hilliard equation, J. Theoret. Probab. 17 (2004), no. 1, 1-49. MR2054575 (2005f:60136)

[2] G. Da Prato, J. Zabczyk, Ergodicity for infinite-dimensional systems, London Mathematical Society Lecture Note Series, 229. Cambridge University Press, Cambridge, 1996. MR:1417491 (97k:60165) 
[3] L. Debbi, On some properties of a high order fractional differential operator which is not in general selfadjoint, Appl. Math. Sci. (Ruse) 1 (2007), no. 25-28, 1325-1339. MR2354419 (2008f:26007)

[4] L. Debbi, M. Dozzi, On the solutions of nonlinear stochastic fractional partial differential equations in one spatial dimension, Stochastic Process. Appl. 115 (2005), no. 11, 1764-1781. MR2172885 (2006h:60107)

[5] T. Funaki, Random motion of strings and related stochastic evolution equations, Nagoya Math. J. 89 (1983), 129-193. MR692348 (85g:60063)

[6] T. Funaki, Regularity properties for stochastic partial differential equations of parabolic type, Osaka J. Math. 28 (1991), no. 3, 495-516. MR.1144470 (93e:60118)

[7] I. Gyöngy, D. Nualart, On the stochastic Burgers' equation in the real line, Ann. Probab. 27 (1999), no. 2, 782-802. MR 1698967 (2000f:60091)

[8] T. Komatsu, On the martingale problem for generators of stable processes with perturbations, Osaka J. Math. 21 (1984), 113-132. MR736974 (86e:60060)

[9] C. Mueller, The heat equation with Lévy noise, Stochastic Process. Appl. 74 (1998), no. 1, 67-82. MR 1624088 (99g:60107)

[10] S. Peszat, J. Zabczyk, Stochastic partial differential equations with Lévy noise. An evolution equation approach, Encyclopedia of Mathematics and its Applications, 113. Cambridge University Press, Cambridge, 2007. MR2356959 (2009b:60200)

[11] T. Shiga, Two contrasting properties of solutions for one-dimensional stochastic partial differential equations, Canad. J. Math. 46 (2) (1994), 415-437. MR.1271224 (95h:60099)

[12] A. Truman, J. L. Wu, On a stochastic nonlinear equation arising from $1 D$ integrodifferential scalar conservation laws, J. Funct. Anal. 238 (2006), no. 2, 612-635. MR.2253735 (2008b:60139)

[13] J. B. Walsh, An introduction to stochastic partial differential equations, École d'eté de probabilités de Saint-Flour, XIV - 1984, pp. 265-439, Lect. Notes Math., 1180. Springer, Berlin, 1986. MR876085 (88a:60114)

[14] B. Xie, The stochastic parabolic partial differential equation with non-Lipschitz coefficients on the unbounded domain, J. Math. Anal. Appl. 339 (2008), no. 1, 705-718. MR2370687 (2009b:60203)

Department of Mathematics and Mechanics, Beijing University of Science and Technology, Beijing, 100083, People's Republic of China

E-mail address: niuminfly@sohu.com

International Young Researchers Emponerment Center and Department of Mathematical Sciences, Faculty of Science, Shinshu University, 3-1-1 Asahi, Matsumoto, NAGANO 390-8621, JAPAN

E-mail address: bxie@shinshu-u.ac.jp, bxie05@sohu.com 